\title{
INSTRUMENTATION OF NUCLEAR REACTORS
}

$\mathrm{A}^{\mathrm{T}}$ $T$ a general meeting of the Institution of Electrical Engineers, held in the Institution's Lecture Theatre on January 8, a symposium of papers on the instrumentation of nuclear reactors was organized, at which about five hundred members and visitors attended, including guests from nuclear energy projects in France, Holland, Sweden, Norway and Italy. Colonel B. H. Leeson, president of the Institution, took the chair and introduced Sir John Cockcroft, whose lecture on "Nuclear Reactors and their Applications" provided a general background for the three specialized papers which followed.

Sir John began by describing the fission process, in which interaction between a neutron and a uranium atom may result in absorption of the former followed by fission of the latter, accompanied by the liberation of further neutrons and the release of energy. The probability of occurrence of this reaction depends upon which isotope of uranium is concerned, and upon the energy of the incident neutron. A system designed to enhance this probability is known as a nuclear reactor, and a classification into three main types is possible: these are known as fast, intermediate and thermal reactors, according to the energy-level at which it is intended that the incident neutron will cause fission. The critical state of a nuclear reactor occurs when, out of the neutrons released in an average fission, one survives all the processes leading to neutron waste, and initiates a further fission. If, however, more than one survives, a cumulatively increasing or divergent reaction results. If the number surviving is smaller than unity, the reaction diminishes until the fissions occur at a rate proportional to their rate of spontaneous occurrence in an isolated sample of uranium. The number of survivors thus defined is known as the reproduction factor, denoted by the symbol $K$; while the reactivity is denoted by $(\delta K)=K-1$.

Fast-fission reactors require a predominating proportion of the uranium-235 isotope in their design, as the 238 isotope can absorb high-energy ncutrons quite strongly without fission occurring. Thermal reactors overcome this difficulty by surrounding the uranium in which the fission occurs by a moderator, the function of which is to absorb the energy of the neutron in a series of scattering collisions. Neutrons of thermal energy (about a fortieth of an electronvolt) are only slightly absorbed by uranium-238. The size of such a reactor is quite large, the mass of 'natural' uranium in the Harwell British Experimental Pile known as BEPO being forty tons and the mass of moderator (graphite in this case) eight hundred tons; 'natural' uranium occurs in the proportions 140 parts of uranium-238 to 1 part of uranium-235. The critical size of a thermal neutron reactor may be reduced by artificially increasing the proportion of uranium-235 present in the uranium, and this sub-type of thermal reactor is known as an enriched reactor.

The third reactor-type employs a neutron energy for causing fission intermediate between fast and thermal, that is, some moderator is used. This is known as an intermediate reactor, and is likely to have importance for mobile nuclear power-plants.

The most important characteristic of a reactor is its neutron balance. A typical balance-sheet for a graphite-moderated thermal neutron reactor is as follows :

Neutrons produced in fission of uranium-235 and in fast fission $2 \cdot 56$

Neutrons carrying on the chain reaction

Neutrons absorbed in uranium-238 to produce plutonium-239 1 Nettrons absorbed in uranium-235 to produce uranilum-236 $0 \cdot 2$

Neutrons absorbed in moderator 0

Neutrons absorbed in structural materials 0.05

Neutrons escaping from core

$0 \cdot(12$

'I'emperatures and neutron-absorbing properties of fission residues affect this balance-sheet and adequate excess $K$ must be allowed for. Since, however, steadystate operation means that $K=1$, control rods have to be provided to absorb any excess of neutrons not required at any particular time.

The plutonium-239 mentioned on the balance sheet can be split by slow neutrons, so that this provides a means of utilizing some of the uranium-238 in addition to the uranium-235. An ideal reactor would produce more secondary fuol (that is, plutonium-239) than primary fuel burnt (that is, uranium-235). With such a 'breeder' reactor we could expect to burn up a great part of the uranium238.

Having briefly surveyed the principles of nuclear reactor operation, Sir John Cockcroft then discussed some of the technical problems of reactors, under the four groupings of physical, metallurgical, chemical and engineering. The physicists are responsible for tho calculations of critical size, supplemented and checked by 'exponential experiments' on a sub-critical reacting-core. They must also determine the adequacy of the means of control and the shielding required. Metallurgists are concerned with the preparation of the uranium fuel elernents. Due to the peculiar lattice-structure of uranium, serious distortion may be caused by thermal cycling and exposure to radiation. The uranium is sheathed in a suitablo metal to seal it completely, and this metal must resist radioactive irradiations, not absorb too many neutrons and withstand the temperatures required by the engine $\theta$ rs. Chemical reactions may be accelerated under irradiation, and chemists are required to study corrosion effects between reactor materials. The engineers have to weave together all these components into a feasible design, including the heattransfer arrangements, the provision of adequate cooling in all circumstances, control and safety.

Turning next to reactors for producing power, Sir John distinguished between the production of substantial quantities of economic power and the development of specialized power-units, for example, for ship propulsion. The first stage in achieving the former target might well be the construction of thermal neutron reactors using natural uranium as a fuel, and either heavy water or graphite as a moderator. Design studies of this type of plant indicate that sizeable blocks of power could be generated at 20-25 per cent efficiency and that, making the conservative assumption that the capital cost of the nuclear station would be twice that of its coal-fired counterpart, and taking realistic figures for the cost of uranium, the cost of the power generated would not be greatly different from conventional practice. 
Sir John stressed that, no credit is allowed in the above figures for the value of the residual plutonium. If, for example, of the uranium-235, one-third were consumed, or $2.3 \mathrm{kgm}$./ton, the reactor would have produced $1.5-2 \mathrm{kgm}$. of plutonium per ton. This would then be available as a basic fuel for the second stage of the power development programme, the breeder reactor stage.

The first approach to breeder reactors will be to develop fast-fission reactors for this purpose, taking advantage of the greatly reduced absorption of fast neutrons by the materials of construction in reactors, and probably using plutonium as the fuel because its average neutron release is nearer 3 than $2 \cdot 55$. An experimental power-producing fast reactor has been working for some time in the United States and made up of a core, "about the size of a regulation football", consisting of uranium with a very high proportion of uranium-235 canned in some suitable metal. The heat developed by the burning of the uranium-235 is removed by the circulation of liquid sodium-potassium alloy. The liquid metal passes through a heat exchanger, and steam is developed to drive a $250-\mathrm{kW}$. power unit. It has not been made public whether breeding has been achieved, although it is said to be "a promising youngster".

Sir John next reviewed the contribution of nuclear power to the resources of world energy, taking his figures (which are in $10^{18} \mathrm{~B}$.Th.U.) mainly from the recent report of the Materials Policy Commission to the President of the United States: the present world annual output of energy is 0.2 ; the world reserves of coal are United States $7 \cdot 6$, Canada 2, United Kingdom 1, China 6, U.S.S.R. 10 and others 6 , the whole totalling 33 ; and the total world reserves of oil are, proved 1 and unproved $4 \cdot 6$, totalling $5 \cdot 6$. The same report estimates that about 25 million tons of uranium are available if we are prepared to pay 100 dollars per $1 b$. of uranium in the ore. If breeding and 100 per cent utilization of uranium were achieved, this would add $1,700 \times$ $10^{18}$ B.Th.U. to world reserves. If we were prepared to pay only 50 dollars/lb., the available reserves would be $250 \times 10^{18}$ B.Th.U. Even at the higher figures, the primary fuel cost would be negligible if breeding were successful. This can be seen at once, since, on these assumptions, the needs of the United Kingdom can be met by 10-15 tons uranium a year at a primary fuel cost of less than 10 million dollars.

In conclusion, Sir John said that the time is not far off when nuclear power using natural uranium fuel will become feasible. These plants would work at reasonable efficiency and would produce power probably at a cost not very much more than from conventional power stations. This would seem to be sufficient to ask from the first experimental units, as all experience shows that costs may be expected to come down as experience accumulates.

Sir John Cockcroft was followed by Mr. R. V. Moore, who introduced his paper on "The Control of a Thermal Neutron Reactor'. 'The operating characteristics of such a reactor were described, both on theoretical grounds and in the light of five years of operation of the Harwell reactors. The importance of the phenomenon of delayed neutrons on the controllability of tho reactor was emphasized, and the following self-induced changes in the reactivity of the system were described: fuel depletion due to burning of uranium-235; poisoning due to the accumulation (owing to fission of uranium) of elements near the centre of the periodic table, certain of these elements (notably xenon-135) being powerful neutron-absorbers; and effects of temperature on reactivity.

The large thermal capacity inevitable with this type of reactor was mentioned, being illustrated by temperature-time curves for BEPO. The selfstabilizing phenomena of thermal reactors wore described- that is, if the reactivity is increased for any reason so that the pile becomes divergent, by suitable design the reaction can be made to terminate itsolf. 'This is because the temperature coefficients are negative-namely, increased temperature involves a self-induced diminution of reactivity. Conditions at starting up and shutting down a reactor were described, as special safety considerations then apply. When starting up, there is an interval during which the neutron density is being raised from an infinitesimal level, which normally applies in the sub-critical state, to the lowest level that the installed instruments are capable of measuring. It is important that the reactor should arrive at this lowest detectable level still diverging sufficiently slowly to enable control to be established. If the reactor has been allowed to acquire excessive reactivity during this 'blind' period, the rate of divergence might be dangerous. On the other hand, advanced types of thermal reactor must be equipped with considerable potential reactivity to counteract the self-induced changes already mentioned. It was Mr. Moore's suggestion that this difficulty could be safely resolved by limiting the rate at which the reactivity might be increased. By studying the self-induced changes, a rate of withdrawal of the control rod may be determined which is normally adequate to compensate these, but which avoids the dangerous start-up condition. At shutdown the difficulty arises that the nuclear reaction cannot be completely cut off at will. This implies that adequate cooling to balance the residual heating must always be available.

The paper concluded by postulating various fault conditions and examining their consequences, and it was Mr. Moore's conclusion that a system of control for thermal nuclear reactors could be evolved which would be inherently safe.

Mr. J. H. Bowen then introduced his paper on "Autornatic Control Characteristics of ThermalNeutron Reactors". From an operating point of view, it is a convenience to abolish all consideration of the rather complex relation between the variation of reactivity and the subsequent variation of power, by installing an automatic power-controller. This device measures the instantaneous reactor power-level and adjusts the reactivity to bring the power automatically to some predetermined or desired level. The differential equations describing the operation of this device include terms involving the response of the reactor to variation of reactivity. These terms, described in the paper as the reactor "transfer function", have been obtained analytically and confirmed by experiment. Typical complete controlsystem responses have been calculated and checked by experiment, with good agreement, and a design method for reactor control-systems was suggested.

The final paper, "Neutron Detectors for Reactor Instrumentation", by 'T. A. J. Jaques, H. A. Ballinger and F. Wade, was introduced by Mr. Jaques. It discussed the principles involved in the detection of thermal neutrons and described two new types of detector recently developed in connexion with the operation of thermal reactors. The first is mainly used as a flux-scanning detector -that is, for local 
measurements of flux density-and is essentially a thermopile with its hot and cold junctions placed adjacent to each other to share equal ambient temperature conditions. One set of junctions, however, is embedded in boron. Under neutron irradiation, the boron - neutron reaction causes unbalanced heating of the junctions, and the instrument thus develops a thermal E.M.F. proportional to the neutron flux density. The response time-constant is of the order of ten seconds.

The second new type is an ionization chamber. These chambers are normally slightly sensitive to incident gamma-ray radiation, in addition to the intended response to neutron flux. This effect has some importance when the reactor is operating at low power following conditions of high-power running, since the residual gamma-radiation may then be comparatively intense whereas the neutron flux is weak. The new chambers are intended partially to overcome this difficulty by measuring separately the gamma-radiation and the neutron-plus-gammaradiation. The former measurement is then automatically subtracted from the latter to leave the quantity required.

In opening the discussion which followed these papers, Dr. Dennis Taylor suggested the use of pulse counters at start-up. These could best be used in conjunction with a logarithmic rate-meter, and the possibility then exists of obtaining directly a measure of the pile reactivity. A period-meter or reactivitymeter of this sort is under development at Harwell. When considering extending the range of power measurements, an inevitable question which arises is whether or not electrometor valves can be considered sufficiently reliable for important applications. In this connexion Dr. Taylor reported the experience gained on BEPO : in the past two years not a single failure due to electrometer valves has been recorded in the log-book.

Dr. A. Lundby (Norway) stated that, in the operation of a heavy-water thermal reactor, photoemission of neutrons from deuterium by gammaradiation has some important effects on reactor control. He believed that the gamma-radiation mainly responsible for this effect is due to the reaction $\mathrm{Ba} \rightarrow{ }^{140} \mathrm{La}$ which has a period of $12 \cdot 8$ days.

$\mathrm{Mr}$. R. Westergaard (Sweden) suggested that Mr. Bowen's paper would be improved by the consideration of the effects of time delays at various points in the system. In particular, he had in mind the use of pulse counters or rate-meters for power measurements at low flux, as this provides an excellent method of discriminating against gammaradiation. But the statistical fluctuations involved in such measurements can only be reduced by rather long integrating-times at the rate-meter, and this must involve corresponding time delays in the automatic-control loop.

Referring to Section 8 of Mr. Moore's paper, M. M. Surdin (France) said that it is the practice to start up the French reactors in the manner suggested, namely, by slowly withdrawing the control rods at constant speed. This speed has been chosen so that the maximum reactivity during the starting transient will not exceed the arbitrary value of 0.3 per cent. This speed corresponds to a rate of reactivity increase of $10^{-5}$ per sec. In this connexion he referred to a calculation by M. M. Ravievski which showed that, when starting up in this manner, the power at which the eritical condition is reached depends only on the rate of reactivity increase. (It may be noted here that similar calculations by $\mathrm{J}$. Woodrow, of Harwell, show that this power is also proportional to the spontaneous or starting power in the shutdown state.)

Mr. W. Kealy (Department of Atomic Energy) made the comment that a reactor entirely devoted to isotope production would be controlled to work continuously at maximum power. This would be limited by temperature considerations, and it is found in practice that fluctuations in the frequency of the electricity supply are sufficient to vary these temperatures by as much as 6 per cent. The system of automatic power control described in Mr. Bowen's paper has to be supplemented by a temperaturesensitive control which, in fact, adjusts the set point of the power controller.

Reference was made by Mr. L. C. Ludbrook (British Thomson-Houston Co., Ltd.) to a few experiments he has made in connexion with the design of a reactor-power controller. These lead to a reactor transfer function in close agreement with Mr. Bowen's, although the approach has been entirely different.

Mr. s. A. Ghalib (Metropolitan-Vickers Electrical Co., Ltd.) said that, in addition to the corrosion problems mentioned by Sir John Cockcroft, a further difficulty for engineers in reactor design work is the problem of friction and wear in these mechanisms, which have to function, often without lubrication and maintenance, in the gas chosen as the reactor coolant. Another point concerns the range of the level of reactor power, quoted in Mr. Moore's paper as $10^{14}$. This obviously applies between the shutdown and full-power states, but Mr. Ghalib suggested that in normal power-producing operations a range of 20 to 1 would be more probable.

\section{CYTOCHROME OXIDASE AND THE 'PASTEUR ENZYME'}

\section{By Prof. D. KEILIN, F.R.S., and Dr. E. F. HARTREE Molteno Institute, University of Cambridge}

TT was shown in 1925 that the multibanded absorpItion spectrum of cytochrome belongs to three hæmochromogen-like components, denoted as cytochromes $a, b$ and $c$, the oxidation and reduction of which-that is, their catalytic activity in respiration - can easily be observed in living intact cells ${ }^{1}$. However, further spectroscopic studies ${ }^{2}$ of the activity of a typical cytochrome system in heart muscle preparations or in suspensions of certain micro-organisms in the presence of different substrates and inhibitors revealed that the absorption bands, at about $605 \mathrm{~m} \mu$ and $448 \mathrm{~m} \mu$ ( $\alpha$-and $\gamma$-bands), do not belong solely to cytochrome $a$ but represent the superimposed and fused bands of two cytochrome components $a$ and $a_{3}$. Cytochrome $a_{3}$, unlike cytochromes $a, b$ and $c$, is very autoxidizable and combines reversibly with respiratory inhibitors such as cyanide, azide, hydrosulphide and carbon monoxide. These properties of cytochrome $a_{3}$, together with the fact that the absorption bands of its carbon monoxide derivative $(\alpha 590 \mathrm{~m} \mu$, and $\gamma 432 \mathrm{~m} \mu)$ occupy approximately the same position as the $\alpha$-and $\gamma$-bands in the photochemical absorption spectrum of yeast ${ }^{3,4}$, enabled us ${ }^{2}$ to identify it with cytochrome oxidase or Warburg's "respiratory ferment". Cytochromes $a$ 